\title{
Interaction between a screw dislocation and stacking faults in FCC metals
}

\author{
Haojie Wei* ${ }^{*}$ Yueguang Wei ${ }^{1}$ \\ State Key Laboratory of Nonlinear Mechanics, Institute of Mechanics, Chinese Academy of Sciences, Beijing 100190, People's Republic of China
}

\section{A R T I C L E I N F O}

\section{Article history:}

Received 4 September 2011

Received in revised form

16 December 2011

Accepted 24 January 2012

Available online 2 February 2012

\section{Keywords:}

Molecular dynamics simulation

Stacking fault

Dislocation

Planar fault energy

Plastic deformation

\begin{abstract}
A B S T R A C T
The mechanical properties of polycrystalline metals greatly depend on the interaction between lattice dislocation and planar defects, but some details about this interaction still need to be explored. In this paper, we investigated the impingement of a screw dislocation on intrinsic stacking fault (SF) and extrinsic stacking faults (ESF) in different FCC metals by using the molecular dynamics simulations. As a screw dislocation approaches a SF from one side, the response of SF will be one of the results: (1) annihilated; (2) transformed into ESF; (3) penetrated. Also, as a screw dislocation interacts with ESF, the response of ESF will be one of the results: (1) transformed into a three-layer thick twin; (2) into an SF; (3) penetrated. Dimensionless parameters relevant to the planar fault energies and their ratios are used to predict the propensity to different interaction modes. The results reveal the evolution law of internal defects clearly, which will be significant to further understand the details of plastic deformation and to seek the underlying techniques for strengthening metals.
\end{abstract}

(C) 2012 Elsevier B.V. All rights reserved.

\section{Introduction}

Previous studies [1,2] have revealed that the mechanical properties of metals, especially in nanoscale, depend tremendously on the generation of, and interactions among, internal defects. Such defects include dislocation, grain boundary, stacking fault, etc. Among these defects, dislocation plays a key role in improving mechanical properties of materials: strengthening consists in the blockage to dislocations, while the ductility consists in the mobility of dislocations [1,2].

Because of the inevitability for dislocations reacting with other defects during deformation [2-4], interactions between dislocations and other defects have always been the hot topics in material science [5-8]. For the strengthening mechanism resulted from the obstruction to dislocations, studies on dislocation interacting with planar defects, especially grain boundaries and coherent twins in FCC metals, have attracted a great deal of interests [9-13].

As one sort of the basic planar defects, stacking faults are commonly observed during synthesis and deformation process in FCC metals [1,14-16]. In FCC structures, within the closest-packed plane, the $\left\{\begin{array}{lll}1 & 1 & 1\end{array}\right\}$-type plane, three equivalent shifted positions are available for the occupation of lattice atoms, referred to as A, B, C layers. The perfect FCC metals cycle among these three layers, with the stacking sequence ...ABCABCABC.... Stacking fault is a planar defect, in which the stacking sequence is locally disturbed by the removal or introduction of a lattice plane. Two types of stacking

\footnotetext{
* Corresponding author. Fax: +861062561284.

E-mail address: weihaojiemail@gmail.com (H. Wei).

1 E-mail address: ywei@LNM.imech.ac.cn (Y. Wei).
}

fault are possible in FCC metals, intrinsic and extrinsic. The stacking sequence of SF is . . ABCABABC. . , while it is . . ABCACBCABC. . . for ESF. The presence of stacking faults will affect the yield strength, creep behavior [17,18] and severe plastic deformation [19]. However, investigations on dislocation-stacking fault interactions are seldom. The interaction between dislocation and extrinsic stacking fault (ESF) for $\mathrm{Al} \mathrm{[20]} \mathrm{and} \mathrm{the} \mathrm{interaction} \mathrm{between} \mathrm{dislocation}$ and stacking fault tetrahedron for $\mathrm{Cu}$ [21-24] have been studied. As to the interactions between dislocation and SF/ESF, there still exist some crucial problems needed to be further investigated. What will happen when a dislocation approaches a SF/ESF? Will the SF/ESF be annihilated? Will the dislocation penetrate the SF/ESF or be blocked? ... All of these questions are fundamental issues to plastic deformation of metals and need to be systematically studied.

Similar to dislocation-twin interaction discussed by Zhu [25], four types of dislocations are available in dislocation-stacking fault interaction. Investigations on the dislocation-twin interaction have revealed that screw dislocation can cross slip into the twin boundary plane to cause twin growth or de-twinning. In order to confirm the possibility of similar dislocation reactions at stacking faults, we focus our attention on a lattice screw dislocation. In the present study, we intend to investigate the interaction between an incoming lattice screw dislocation and a pre-existing SF/ESF by using the molecular dynamics (MD) simulations. Although the strengthening roles of stacking faults may be less abundant than grain boundaries and coherent twins, it is hoped that a profound comprehension of dislocation-SF/ESF interaction will contribute to reveal the evolution of internal defects, to further understand the details of plastic deformation and to seek the underlying techniques for strengthening metals. 


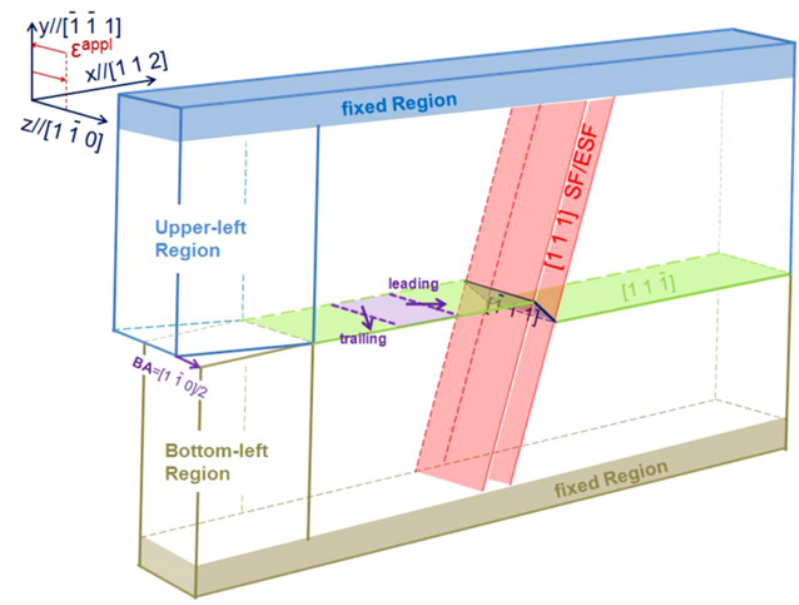

Fig. 1. MD configuration for simulating the interaction between an incoming screw

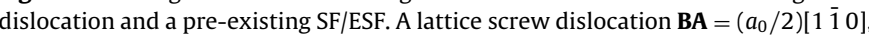
created via relative rigid displacements of the upper-left and bottom-left regions of the MD cell (see Refs. $[9,10]$ ) and driven by a constant shear strain $\varepsilon^{\text {appl }}$, approaches the SF/ESF. Several layers of atoms on the top and bottom are fixed. The pink planes indicate the two hcp planes of SF or ESF, while the green planes illustrate the [1 $11 \overline{1}$ glide plane on both sides of SF/ESF. The violet lines with a ribbon between them indicate the extended dislocation, dissociated from the lattice screw dislocation.

\section{Simulation methods}

MD simulations are carried out within a rectangular block with a pre-existing SF/ESF. The orientations of three axes are shown in Fig. 1. We adopt a computational cell containing 145,000 atoms with the edges $100\left[\begin{array}{lll}1 & 1 & 2\end{array}\right], 40\left[\begin{array}{lll}\overline{1} & \overline{1} & 1\end{array}\right]$ and $3\left[\begin{array}{lll}1 & \overline{1} & 0\end{array}\right]$, replicated periodically in all three directions. Periodic boundary condition is applied in $z$-axis. Several outermost layers of atoms in $y$-axis are fixed. Rigid displacement $[9,10]$ is used to create the incoming lattice screw dislocation $\mathbf{B A}=a_{0} / 2\left[\begin{array}{lll}1 & \overline{1} & 0\end{array}\right]$.

In our simulations, the dislocations are of straight with "infinite" length due to the periodic boundary condition applied along the dislocation line direction. The SF/ESF planes are also infinite in the same direction.

In order to systematically study the interaction between a screw dislocation and SF/ESF, five FCC metals $\mathrm{Al}, \mathrm{Au}, \mathrm{Ni}, \mathrm{Cu}$ and $\mathrm{Ag}$ are used. Grochola's EAM potential for Au [26], Williams' EAM potential for $\mathrm{Ag}$ [27], and Mishin's EAM potentials for $\mathrm{Al}$ [28], $\mathrm{Ni}$ [28] and $\mathrm{Cu}$ [29] are adopt in our simulations. For each metal, different shear strains $\left(\varepsilon^{\mathrm{appl}}\right)$ are applied to investigate the interaction modes in variable stress states. MD simulations for different shear-strain loadings are independent of each other.

All of the simulations start from near absolute zero $(0.1 \mathrm{~K})$ and the thermal fluctuation is neglected. At the beginning of the MD simulation, a constant shear strain $\varepsilon^{\text {appl }}$ is applied to the whole simulation model. Then the model is relaxed governed by the constant energy ensemble.

\section{Results}

\subsection{Dislocation reactions before $S F / E S F$}

According to Frank's dislocation reaction criterion, it is of energetically favorable that once being created, the lattice screw dislocation will dissociate into an extended dislocation, as indicated by Jin et al. [9]:

$$
\begin{array}{ccccc}
\mathbf{B A} & \rightarrow & \gamma \mathbf{A} & + & \mathbf{B} \boldsymbol{\gamma} \\
\frac{1}{2}(11 \overline{1})[1 \overline{1} 0] & \rightarrow & \frac{1}{6}(11 \overline{1})[2 \overline{1} 1] & + & \frac{1}{6}(11 \overline{1})[1 \overline{1} \overline{1}]
\end{array}
$$

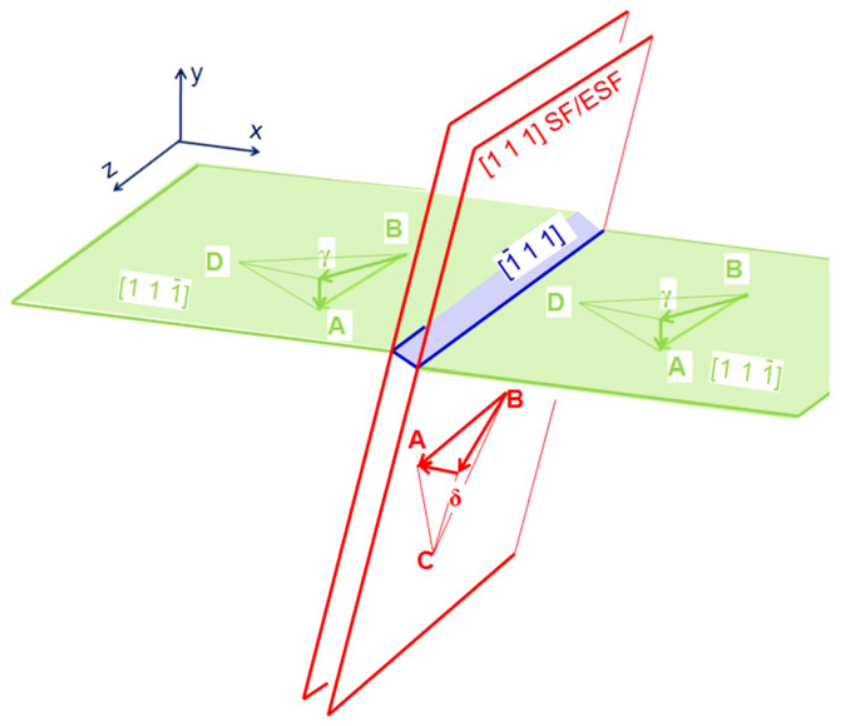

Fig. 2. Illustration of dislocation reactions during the interaction between screw dislocation and SF/ESF. Thompson tetrahedron notation is used. The dislocations are colored the same as their glide planes.

Driven by the externally applied shear strain, the extended dislocation will approach the SF/ESF from one side. However, it cannot penetrate the SF/ESF into the other side for the energy barrier caused by the transition of lattice orientation within the two HCP layers of SF/ESF. Instead, the leading partial will be blocked before the SF/ESF and the trailing partial glide continuously toward the $\mathrm{SF} / \mathrm{ESF}$. The screw dislocation can only be combined into a lattice dislocation if the external stress is sufficient (the dislocation reactions are illustrated in Fig. 2):

$$
\begin{aligned}
& \gamma \mathbf{A}+\mathbf{B} \gamma \quad \rightarrow \quad \text { BA } \\
& \frac{1}{6}(11 \overline{1})[2 \overline{1} 1]+\frac{1}{6}(11 \overline{1})[1 \overline{2} \overline{1}] \rightarrow \frac{1}{2}(11 \overline{1})[1 \overline{1} 0] \\
& 30^{\circ} \text { leading partial }+30^{\circ} \text { trailing partial } \rightarrow \text { lattice dislocation }
\end{aligned}
$$

Otherwise, the screw dislocation will be rejected by the SF/ESF.

After the re-combination of the two partials, the lattice dislocation will react with SF/ESF and new Shockley partials will be emitted, in order to reduce the internal stress concentration resulted from the externally applied shear stress.

\subsection{Interaction between a screw dislocation and SF}

MD simulations reveal that three paths are available for the re-nucleation of Shockley partials, which correspond to the three interaction modes between screw dislocation and SF:

(1) The lattice screw dislocation dissociates into two Shockley partials on an adjacent plane parallel to SF (Eq. (3a)). The SF is transformed into ESF (Fig. 3b).

(2) The lattice screw dislocation dissociates into two Shockley partials, propagating oppositely along the SF plane (Eq. (3b)). The SF is annihilated after the sweep of the newly formed Shockley partials (Fig. 3c).

(3) The lattice screw dislocation penetrates the SF and then redissociates into an extended dislocation on the other side of the SF (Eq. (1)). The glide plane of the newly formed dislocation has a shift of $(2 \sqrt{3} / 9) a_{0}$ relative to the former glide plane (Fig. 3d). 
(a)

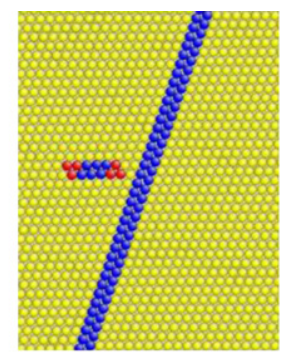

(b)
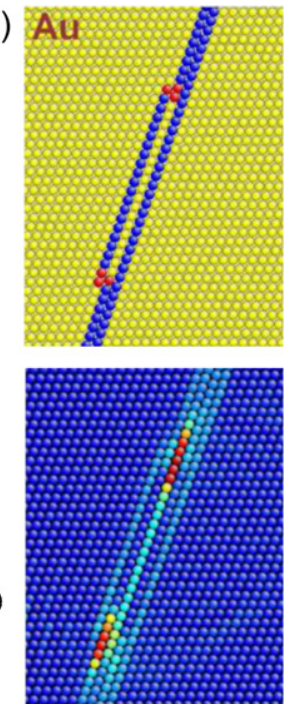

(c)
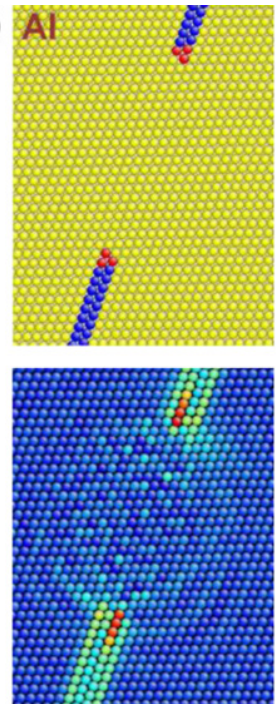

(d)
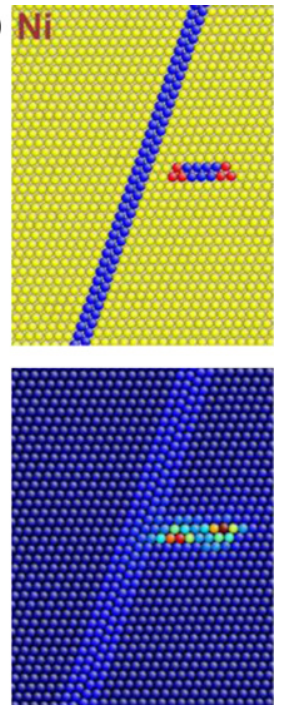

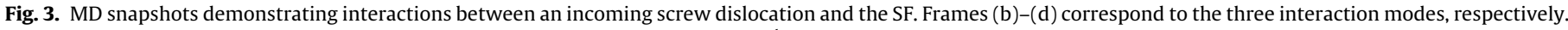

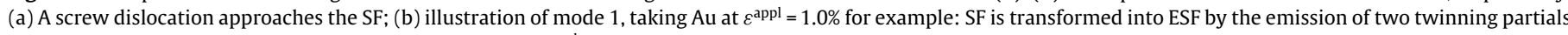

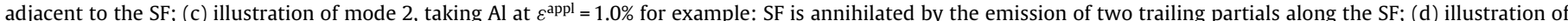

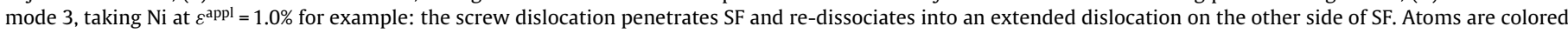
according to the common neighbor analysis (CNA) and atomic potential energy in the upper and lower images, respectively (similarly, in Fig. 4).

$$
\begin{aligned}
& \begin{array}{ccccc}
\mathbf{B A} & \rightarrow & \boldsymbol{\delta A} & + & \mathbf{B} \boldsymbol{\delta} \\
\frac{1}{2}(111)[1 \overline{1} 0] & \rightarrow & \frac{1}{6}(111)[1 \overline{2} 1] & + & \frac{1}{6}(111)[2 \overline{1} \overline{1}]
\end{array} \\
& \text { lattice dislocation } \rightarrow 30^{\circ} \text { twinning partial }+30^{\circ} \text { twinning partial } \\
& \text { lattice dislocation } \rightarrow 30^{\circ} \text { trailing partial }+30^{\circ} \text { trailing partial }
\end{aligned}
$$

The two Shockley partials on the right side of Eq. (3b) are both trailing partials. The reason will be discussed in Section 4.1.2.

The interaction modes for different metals and the range of applied shear strain corresponding to each mode are all summarized in Table 1. As to screw dislocation-SF interaction, Au exhibits only mode 1 ; Al exhibits only mode 2 ; and the other FCC metals simulated exhibit mode 2 in small shear strain and mode 3 in large shear strain.

\subsection{Interaction between a screw dislocation and ESF}

Meanwhile, MD simulations reveal three interaction modes between screw dislocation and ESF:

(4) The screw dislocation dissociates into two Shockley partials adjacent to the first HCP plane of the ESF (Eq. (3a)). The ESF is transformed into a three-layer thick twin (Fig. 4b).

(5) The screw dislocation dissociates into two Shockley partials adjacent to the second HCP plane of the ESF (Eq. (3a)). The ESF is transformed into SF (Fig. 4c).
(6) The screw dislocation penetrates the ESF and re-dissociates into an extended dislocation on the other side of the ESF (Eq. (1)). The newly formed dislocation has a shift of $(4 \sqrt{3} / 9) a_{0}$ relative to the former glide plane (Fig. $4 \mathrm{~d}$ ).

From the summarization in Table 1, Au exhibits only mode 4; $\mathrm{Ni}$ exhibits only mode 6; Al exhibits mode 4 in small shear strain and mode 6 in large shear strain; $\mathrm{Cu}$ and $\mathrm{Ag}$ experience mode 4, mode 5 and mode 6 with the increase of the applied shear strain.

\section{Discussion}

\subsection{Resistances to different interaction modes}

The criterion for dislocation nucleation, based on Peierls concept and introduced by Rice [30], has been widely used to explain dislocation behaviors associated with crack propagation [31,32] and plastic deformation [33] in FCC metals. In the following discussion, we will first analyze the energy barrier encountered by each interaction mode, using the same method as Rice and Tadmor.

\subsubsection{Resistances to mode 1 , mode 4 and mode 5}

In mode 1 (transformation from SF to ESF), mode 4 (transformation from ESF to a three-layer thick twin) and mode 5 (transformation from ESF to SF), the systems are all transformed

Table 1

\begin{tabular}{|c|c|c|c|c|c|c|c|c|c|c|c|c|}
\hline \multirow[t]{3}{*}{ Metal } & \multicolumn{6}{|c|}{ Range of $\varepsilon^{\text {appl }}(\%)$} & \multicolumn{6}{|c|}{ Critical shear strain (\%) and resolved shear stress (GPa) } \\
\hline & \multicolumn{3}{|c|}{ Screw dislocation-SF interaction } & \multicolumn{3}{|c|}{ Screw dislocation-ESF interaction } & \multicolumn{2}{|c|}{$\begin{array}{l}\text { Combination } \\
\text { before SF/ESF }\end{array}$} & \multicolumn{2}{|c|}{ SF penetration } & \multicolumn{2}{|c|}{ ESF penetration } \\
\hline & $\begin{array}{l}\text { Mode } 1 \\
\mathrm{SF} \rightarrow \mathrm{ESF}\end{array}$ & $\begin{array}{l}\text { Mode } 2 \\
\text { annihilation }\end{array}$ & $\begin{array}{l}\text { Mode } 3 \\
\text { penetration }\end{array}$ & $\begin{array}{l}\text { Mode } 4 \\
\text { ESF } \rightarrow \text { Twin3 }\end{array}$ & $\begin{array}{l}\text { Mode } 5 \\
\mathrm{ESF} \rightarrow \mathrm{SF}\end{array}$ & $\begin{array}{l}\text { Mode } 6 \\
\text { penetration }\end{array}$ & Strain & Stress & Strain & Stress & Strain & Stress \\
\hline $\mathrm{Al}$ & & $\geq 0.6$ & & $0.6-3.9$ & & $\geq 4.0$ & 0.6 & 168.0 & & & 4.0 & 1120.0 \\
\hline $\mathrm{Au}$ & $\geq 0.6$ & & & $\geq 0.6$ & & & 0.6 & 156.0 & & & & \\
\hline $\mathrm{Ni}$ & & $0.6-0.9$ & $\geq 1.0$ & & & $\geq 0.6$ & 0.6 & 448.0 & 1.0 & 746.7 & 0.6 & 448.0 \\
\hline $\mathrm{Cu}$ & & $0.7-1.6$ & $\geq 1.7$ & $0.7-1.7$ & 1.8 & $\geq 1.9$ & 0.7 & 288.2 & 1.7 & 699.8 & 1.9 & 782.2 \\
\hline $\mathrm{Ag}$ & & $0.7-1.4$ & $\geq 1.5$ & $0.7-1.2$ & $1.3-1.5$ & $\geq 1.6$ & 0.7 & 179.0 & 1.5 & 383.5 & 1.6 & 409.1 \\
\hline
\end{tabular}

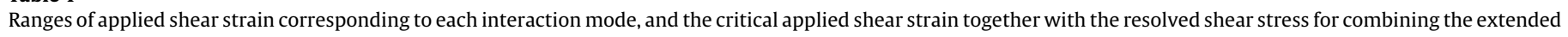

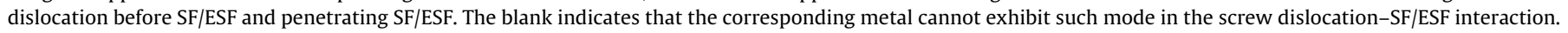


(a)

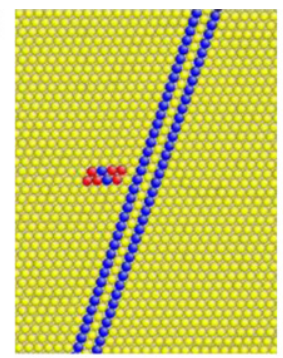

(b)
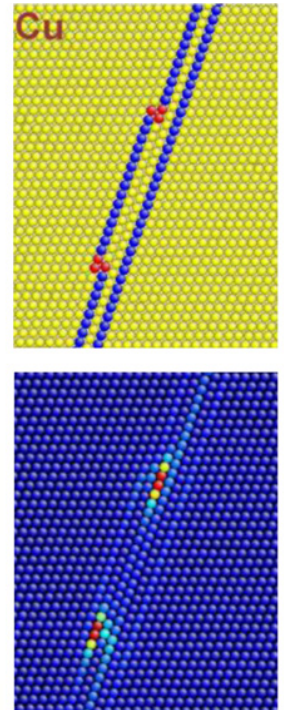

(c)
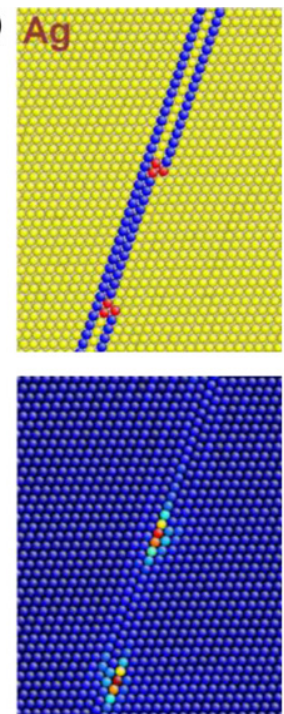

(d) $\mathrm{A}$
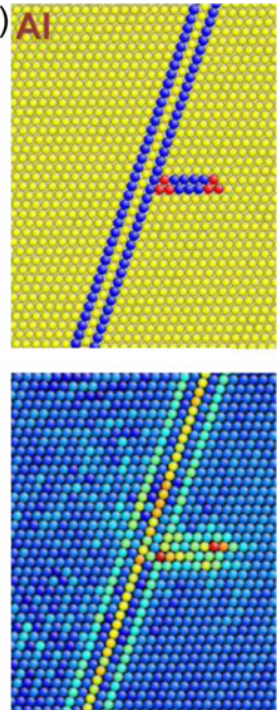

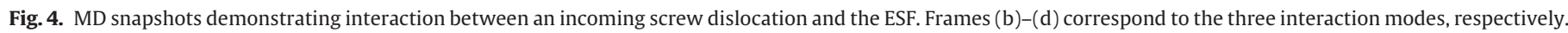

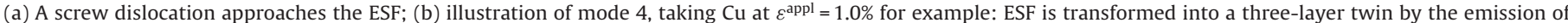

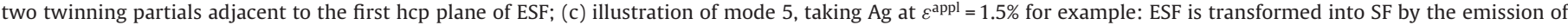

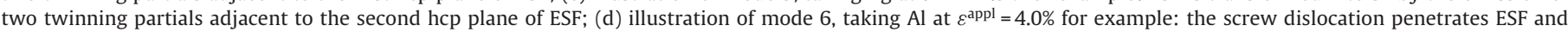
re-dissociates into an extended dislocation on the other side of ESF.

via the emissions of twinning partials, and therefore all of the three transformations can be considered as twin boundary migration. However, for the differences of start configurations among these modes, the energy barriers associated with the emission of twinning partials are $\gamma_{\mathrm{utf}}-\gamma_{\mathrm{sf}}$ for mode $1, \gamma_{\mathrm{ut} 3}-\gamma_{\mathrm{esf}}$ for mode 4 and $\gamma_{\text {utf }}-\gamma_{\text {esf }}$ for mode 5 , respectively. (Where $\gamma_{\text {utf }}$ is the energy difference during the transformation from SF to ESF, $\gamma_{\text {sf }}$ is the stacking fault energy, $\gamma_{\text {ut3 }}$ is the energy difference during the transformation from ESF to a three-layer thick twin, and $\gamma_{\text {esf }}$ is the extrinsic stacking fault energy.)

Due to the accuracy limit of MD simulations based on EAM potentials, the energy differences among all of the three transformations and twin boundary migration are negligible (see Table 2). Therefore the energy barrier to each transformation can be replaced by that of twin boundary migration, which is $\gamma_{\mathrm{utm}}[34,35]$. Thus, all of the three modes can be estimated by a uniform dimensionless parameter $\Pi_{1}=\gamma_{\mathrm{utm}} / \mu b_{\mathrm{S}}$. (Where $\gamma_{\mathrm{utm}}$ is the energy barrier for twin boundary migration; $\mu=\left(C_{11}-C_{12}+C_{44}\right) / 3$ is the shear modulus [30] along the $\{111\}\langle 1 \overline{1} 0\rangle$ shear plane; $C_{11}, C_{12}$ and $C_{44}$ are elastic constants; and $b_{\mathrm{S}}=a_{0} / \sqrt{6}$ is the mode of Burgers vector of Shockley partial.)

\subsubsection{Resistances to mode 2, mode 3 and mode 6}

As shown by MD simulations, after the lattice dislocation penetrates and propagates into the other side of SF/ESF, a Shockley partial will nucleate firstly. Because the Shockley partial firstly nucleating in perfect FCC lattice structure is a leading partial, both of the newly formed Shockley partials in mode 3 (penetrating SF) and mode 6 (penetrating ESF) should be leading partials.

It is known in FCC metals that a SF will be left behind the leading partial and be annihilated after the emission of a trailing partial. That is to say, the annihilation of SF results from the sweep of trailing partial. Thus, the two newly formed partials are both trailing partials in mode 2 (see the right side of Eq. (3b)).

Based on the analysis above, the occurrences of mode 3 (penetrating SF) and mode 6 (penetrating ESF) are both determined by the nucleation of leading partial, while the occurrence of mode 2 (annihilating SF) is determined by the nucleation of trailing partial.

As indicated by Rice [30] and Tadmor [32], for the emission of leading partial, the system will have to pass through an energy barrier which is referred to as the unstable stacking fault energy $\gamma_{\text {usf }}$; while the energy barrier encountered by the emission of trailing partial is the energy difference between stacking fault and unstable stacking fault, $\gamma_{\text {usf }}-\gamma_{\text {sf }}$. Consequently, the resistance to modes 3 and 6 can be estimated by a dimensionless parameter $\Pi_{3}=\gamma_{\mathrm{usf}} / \mu b_{\mathrm{S}}$, and the resistance to mode 2 can be estimated by another dimensionless parameter $\Pi_{2}=\left(\gamma_{\mathrm{usf}}-\gamma_{\mathrm{sf}}\right) / \mu b_{\mathrm{S}}$. (Where $\gamma_{\text {usf }}$ is the unstable stacking fault energy.)

\subsection{Explanation of screw dislocation-SF interaction}

Next, we will discuss the criteria for different interaction modes. The discussions are based on the comparisons of the dimensionless parameters mentioned in Section 4.1. By comparing interaction modes in Table 1 and the corresponding dimensionless parameters in Table 2, we can find the criterion for each mode. The planar fault energies $\gamma_{\text {usf }}, \gamma_{\mathrm{sf}}, \gamma_{\mathrm{utf}}, \gamma_{\mathrm{esf}}, \gamma_{\mathrm{ut} 3}, \gamma_{\mathrm{t} 3}$ and $\gamma_{\mathrm{utm}}$ are all calculated via standard method [36] by rigid shifting two semi-infinite blocks of crystal along the six paths illustrated in Fig. 5. The material constants, planar fault energies, dimensionless parameters and their ratios calculated for the five FCC metals are all summarized in Table 2.

The three interaction modes between screw dislocation and SF correspond to three types of dislocation nucleation. If twinning partials nucleate, the SF is transformed into ESF; if trailing partials do, the SF is annihilated; and the SF is penetrated if leading partial nucleate on the other side of SF. We will expound the dislocation behaviors in screw dislocation-SF interactions from two aspects: one is the competition between mode 1 and mode 2 ; the other is the competition between mode 2 and mode 3 .

\subsubsection{Competition between mode 1 and mode 2}

In mode 1 and mode 2 , the newly formed Shockley partials glide both along the plane parallel to SF, but they nucleate from different sites. The Shockley partials nucleate from the plane adjacent to SF in the former, while the partials nucleate just along SF in the latter.

Whether the SF will be transformed into ESF (mode 1) or be annihilated (mode 2 ) depends on the competition between trailing partial nucleation and twinning partial nucleation. This 
Table 2

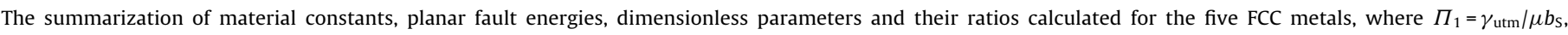
$\Pi_{2}=\left(\gamma_{\mathrm{usf}}-\gamma_{\mathrm{sf}}\right) / \mu b_{\mathrm{s}}, \Pi_{3}=\gamma_{\mathrm{usf}} / \mu b_{\mathrm{s}}, \Pi_{1} / \Pi_{2}=\gamma_{\mathrm{utm}} /\left(\gamma_{\mathrm{usf}}-\gamma_{\mathrm{sf}}\right), \Pi_{1} / \Pi_{3}=\gamma_{\mathrm{utm}} / \gamma_{\mathrm{usf}}, \Pi_{2} / \Pi_{3}=\left(\gamma_{\mathrm{usf}}-\gamma_{\mathrm{sf}}\right) / \gamma_{\mathrm{usf}}$.

\begin{tabular}{|c|c|c|c|c|c|c|c|c|c|c|c|c|c|c|c|}
\hline \multirow[t]{2}{*}{ Metal } & \multicolumn{2}{|c|}{ Material constant } & \multicolumn{7}{|c|}{ Fault energy $\left(\mathrm{mJ} \mathrm{m}^{-2}\right)$} & \multicolumn{3}{|c|}{ Dimensionless Parameter } & \multicolumn{3}{|l|}{ Ratio } \\
\hline & $b_{\mathrm{S}}(\AA)$ & $\mu(\mathrm{GPa})$ & $\gamma_{\text {usf }}$ & $\gamma_{\mathrm{sf}}$ & $\gamma_{\text {utf }}$ & $\gamma_{\text {esf }}$ & $\gamma_{\mathrm{ut} 3}$ & $\gamma_{\mathrm{t} 3}$ & $\gamma_{\mathrm{utm}}$ & $\Pi_{1}$ & $\Pi_{2}$ & $\Pi_{3}$ & $\Pi_{1} / \Pi_{2}$ & $\Pi_{2} / \Pi_{3}$ & $\Pi_{1} / \Pi_{3}$ \\
\hline $\mathrm{Al}$ & 1.653 & 28.00 & 168.1 & 145.9 & 220.3 & 150.4 & 221.5 & 150.6 & 70.6 & 0.0152 & 0.0048 & 0.0363 & 3.1816 & 0.1319 & 0.4197 \\
\hline $\mathrm{Au}$ & 1.662 & 26.00 & 92.5 & 42.2 & 101.1 & 42.3 & 100.6 & 42.2 & 58.5 & 0.0135 & 0.0116 & 0.0214 & 1.1632 & 0.5438 & 0.6325 \\
\hline $\mathrm{Ni}$ & 1.437 & 74.67 & 367.3 & 125.3 & 426.9 & 127.0 & 428.6 & 126.9 & 302.1 & 0.0282 & 0.0225 & 0.0342 & 1.2487 & 0.6587 & 0.8225 \\
\hline $\mathrm{Cu}$ & 1.476 & 41.17 & 162.0 & 44.3 & 183.3 & 44.4 & 183.4 & 44.4 & 139.1 & 0.0229 & 0.0194 & 0.0267 & 1.1819 & 0.7264 & 0.8585 \\
\hline $\mathrm{Ag}$ & 1.670 & 25.57 & 114.8 & 17.8 & 123.3 & 18.4 & 123.6 & 18.4 & 105.4 & 0.0247 & 0.0227 & 0.0269 & 1.0874 & 0.8447 & 0.9185 \\
\hline
\end{tabular}

competition can be estimated by the ratio of $\Pi_{1}$ to $\Pi_{2}$, i.e. $\Pi_{1} / \Pi_{2}=\gamma_{\mathrm{utm}} /\left(\gamma_{\mathrm{usf}}-\gamma_{\mathrm{sf}}\right)$. A small value of $\Pi_{1} / \Pi_{2}$ favors the annihilation of SF and makes against of penetration. As listed in Table 2 , the value of $\Pi_{1} / \Pi_{2}$ for $\mathrm{Al}$ (3.1816) is far larger than unity, which means the nucleation of trailing partial is rather easier than the nucleation of twinning partial. The SF in Al can only be annihilated, no matter how large the external stress is.

However, only the relative value of $\Pi_{1} / \Pi_{2}$ is not sufficient to explain the MD results associated with the competition between twinning partial nucleation and trailing partial nucleation. Expect $\mathrm{Al}$, the values of $\Pi_{1} / \Pi_{2}$ for the other simulated metals are rather close to each other. If it is deduced only from the value of $\Pi_{1} / \Pi_{2}$, these four metals should have the similar propensity for dislocation nucleation. However, it is only in Au that the twinning partial nucleates, shown by our MD simulations. If we compare the absolute value of $\Pi_{1}$ for these four metals, we may find that the value of $\Pi_{1}$ for $\mathrm{Au}$ is the smallest and the difference is obvious between $\mathrm{Au}$ (0.0135) and other metals (0.0229-0.0282).

Thus, we can draw the conclusion naturally: it is not only the relative value of $\Pi_{1} / \Pi_{2}$ but also the absolute value of $\Pi_{1}$ that determine whether twinning partials or trailing partials emit. The relative value of $\Pi_{1} / \Pi_{2}$ reflects the twinning propensity in comparison with trailing partial nucleation, while the absolute value of $\Pi_{2}$ reflects the twinning propensity from the physical nature of material. In screw dislocation-SF interaction, twinning partial, rather than trailing partial, can only emits from materials meeting the following conditions: (1) the relative value of $\Pi_{1} / \Pi_{2}$ is close to unity; and (2) the absolute value of $\Pi_{2}$ is smaller than a threshold value, which seems to be some value ranging from 0.0135 to 0.0229 , according to our simulation.
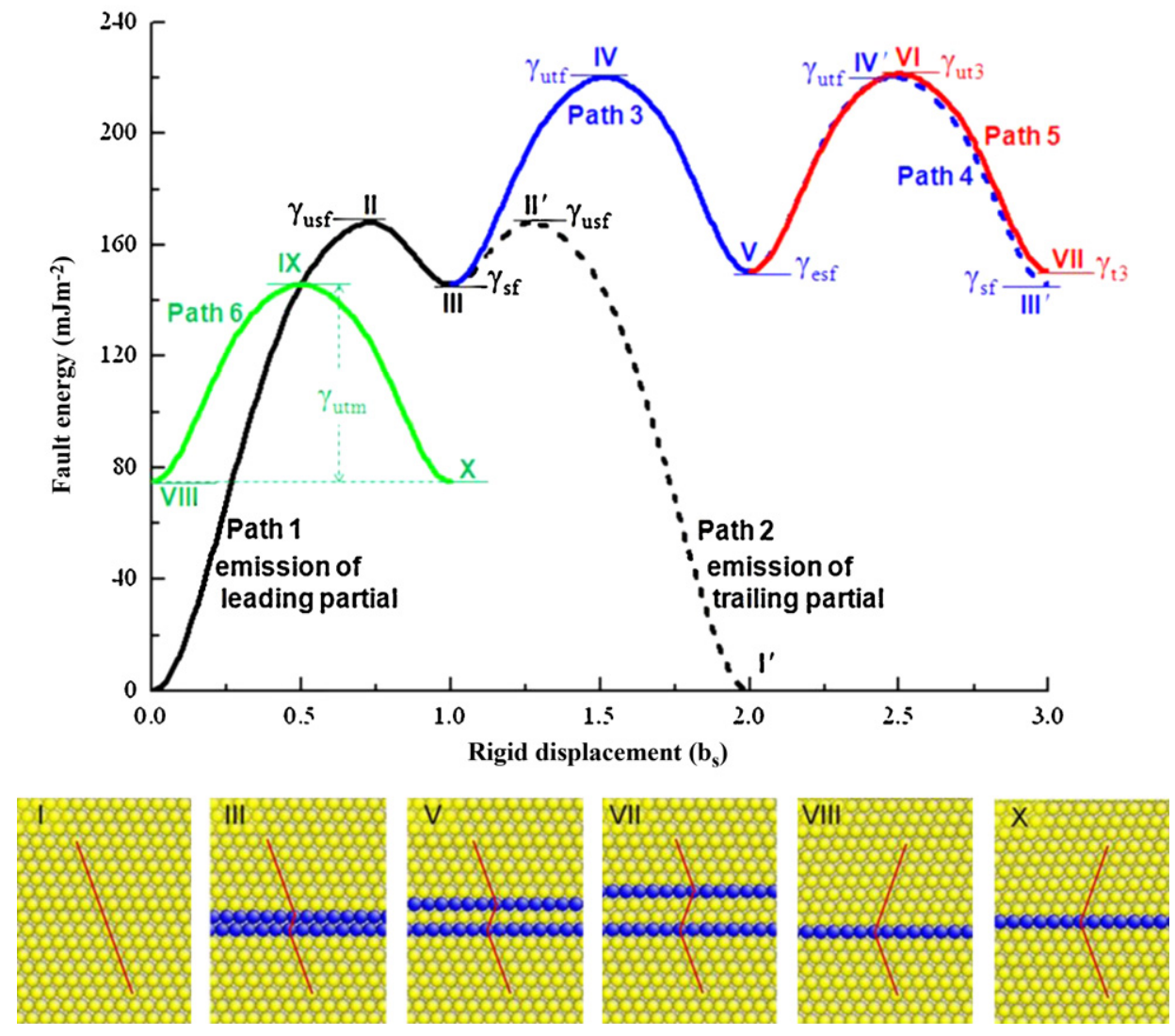

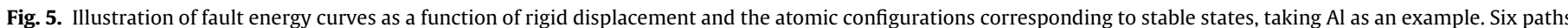

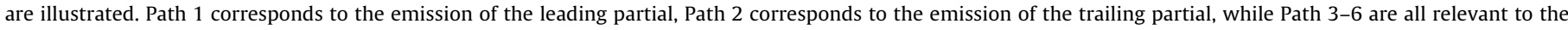

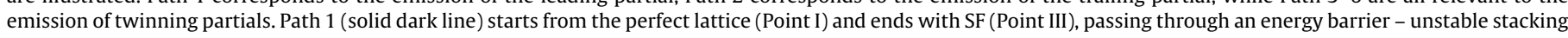

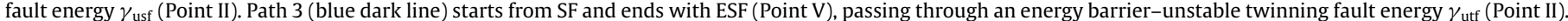

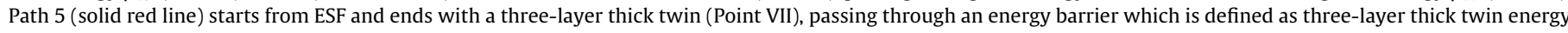

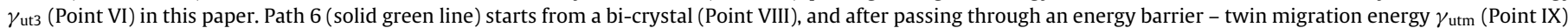

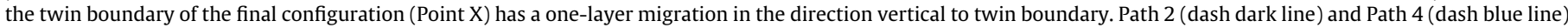

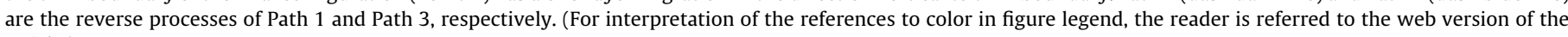
article.) 


\subsubsection{Competition between mode 2 and mode 3}

In mode 2 and mode 3 , the newly formed Shockley partials are entirely different. The Shockley partials nucleate and glide along SF in the former, while the partials nucleate from the other side of SF in the latter.

Whether the SF will be annihilated (mode 2) or be penetrated (mode 3 ) depends on the competition between trailing partial nucleation and leading partial nucleation. The ratio of $\Pi_{2}$ to $\Pi_{3}$, $\Pi_{2} / \Pi_{3}=\left(\gamma_{\mathrm{usf}}-\gamma_{\mathrm{sf}}\right) / \gamma_{\mathrm{usf}}$, reflects such competition.

As listed in Table 2 , the value of $\Pi_{2} / \Pi_{3}$ is less than unity. That means that the nucleation of trailing partial along SF is easier than the nucleation of leading partial on the other side of SF. The smaller the value of $\Pi_{2} / \Pi_{3}$ is, the easier the trailing partial emits. As to Al, owing to the smallest value of $\Pi_{2} / \Pi_{3}(0.1319)$, the emission of the leading partial becomes impossible, and the SF in Al can only be annihilated (mode 2). On the contrary, the SFs in $\mathrm{Ni}, \mathrm{Cu}$ and $\mathrm{Ag}$ are annihilated in small shear strain and penetrated with the aid of a large shear strain, for the values of $\Pi_{2} / \Pi_{3}$ are close to unity and the emissions of leading partials become possible in these metals.

\subsection{Explanation of screw dislocation-ESF interaction}

The two layers of HCP atoms in ESF can be considered as two obstacles to the motion of screw dislocation. Without the obstacles, the screw dislocation can move straight along its former glide plane. Due to the existence of ESF, two paths are available: (1) the dislocation reacts with the ESF, and then the ESF will be transformed into SF or a three-layer thick twin, as shown by MD results; (2) the dislocation penetrates the ESF. Which will be chosen depends on the competition between the resistances encountered by these two paths.

The ratio of $\Pi_{1}$ to $\Pi_{3}$, i.e. $\Pi_{1} / \Pi_{3}=\gamma_{\mathrm{utm}} / \gamma_{\mathrm{usf}}$, can be used to predict such competition. The numerator indicates the resistance to twinning partial nucleation, while the denominator depicts the resistance to leading partial nucleation. A small value of $\Pi_{1} / \Pi_{3}$ favors transformation (from ESF to a three-layer thick twin in mode 4 , or from ESF to SF in mode 5 ), and a large value of $\Pi_{1} / \Pi_{3}$ means the reduction of difficulty in penetration (mode 6 ).

The $\Pi_{1} / \Pi_{3}$ value for $\mathrm{Al}(0.4197)$ is far less than unity, which is also the smallest among the simulated metals, and therefore the resistance to twinning partial nucleation is naturally far less than that to leading partial. Once the external stress is large enough for the recombined lattice dislocation to react with ESF, twinning partials will be nucleated and the ESF will be transformed into a three-layer thick twin (mode 4). The ESF in Al cannot be penetrated until rather large shear strain $(\geq 0.04)$ is applied. Then, with the aid of the increased external stress, dislocation can penetrate the ESF (mode 6), after overcoming the energy barrier caused by ESF.

The value of $\Pi_{1} / \Pi_{3}$ for $\mathrm{Cu}$ and $\mathrm{Ag}$ (0.8585 and 0.9185, respectively) are closer to unity than other metals, which means the resistance to the nucleation of twinning partial is comparable with that to the nucleation of leading partial. Therefore, all of the three interaction modes are available for $\mathrm{Cu}$ and $\mathrm{Ag}$, with the aid of external stress: (1) in the case of little external stress, dislocation is blocked by, and reacts with, the first HCP layer of ESF, twinning partials emit along the plane adjacent the first HCP layer, and the ESF is transformed into a three-layer thick twin (mode 4); (2) when the external stress is large enough to overcome the blockage of the first HCP layer, the dislocation will react with the second HCP layer and the ESF will be transformed into SF (mode 5); (3) if the external stress is sufficient to break through both of the two obstacles caused by ESF, the screw dislocation will penetrate the ESF.

As discussed in screw dislocation-SF interaction, the absolute value of $\Pi_{1}$ also plays an important role in screw dislocation-ESF interaction. For $\mathrm{Ni}$, the value of $\Pi_{1}(0.0282)$ is the largest among the simulated metals, which means the difficulty for twinning partial nucleation is so great that the emission of twinning partial is impossible. So, the ESF in Ni can only be penetrated (mode 6). As for $\mathrm{Au}$, with the least value of $\Pi_{1}(0.0135)$, the twinning propensity is so clear that the lattice dislocation will be blocked by, and the twinning partial will emit adjacent to, the first HCP layer. That is to say, the dislocation will prefer to react with the first HCP layer, not matter how large the external stress is. Therefore, the dislocation cannot penetrate the first HCP layer, let alone the second one. Thus the ESF in Au can only be transformed into a three-layer thick twin (mode 4).

\subsection{Critical shear strain/stress}

There is a critical shear strain $\left(\varepsilon^{\mathrm{appl}}\right) /$ shear stress $\left(\sigma^{\mathrm{appl}}\right)$ for the combination of the extended dislocation before SF/ESF. The critical value is determined by the balance between the attraction caused by the stacking fault and the repulsion caused by the two partials. For each metal, the shear strain/stress required for re-combing the extended dislocation before SF is just the same as that before ESF. These critical values for the simulated metals can be found in Table 1. If $\varepsilon^{\mathrm{appl}} / \sigma^{\mathrm{appl}}$ is less than this threshold value, the screw dislocation will be blocked and rejected by SF/ESF.

The critical shear strain required for the annihilation of SF is the same as that for the combination of extended dislocation. If such mode is available to FCC metal, the trailing partials will emit as soon as the extended dislocation is re-combined.

However, the resolved shear stress (RSS) required for a screw dislocation to annihilate a $\mathrm{SF}, \sigma_{\mathrm{A}}$, is only $1 / 3$ of the applied shear stress, according to $\sigma_{A}=(1 / 3) \sigma^{\mathrm{appl}}=(1 / 3) \mu \varepsilon^{\mathrm{appl}}[9,10]$. In order to annihilate a SF, the RSS should be 56.0 MPa for Al, 149.3 MPa for Ni, 96.1 MPa for $\mathrm{Cu}$ and $59.7 \mathrm{MPa}$ for $\mathrm{Ag}$.

The RSS required to penetrate a SF/ESF, $\sigma_{\mathrm{P}}=\sigma^{\mathrm{appl}}=\mu \varepsilon^{\mathrm{appl}}$, can also be calculated (see Table 1 ). In order to penetrate a SF, the RSS should be 746.7 MPa for Ni, 699.8 MPa for Cu and 383.5 MPa for Ag. The screw dislocation can only penetrate the SF when RSS is larger than this threshold. As for Al, the annihilation of SF is so easy that it is impossible for penetration. In order to penetrate an ESF, RSS should be $1120.0 \mathrm{MPa}$ for $\mathrm{Al}, 448.0 \mathrm{MPa}$ for $\mathrm{Ni}$, 782.2 MPa for $\mathrm{Cu}$ and 409.1 MPa for Ag. The screw dislocation can only penetrate the ESF when RSS is larger than this threshold.

\section{Summary}

In this paper, we theoretically analyze the possible interaction modes between a lattice screw dislocation and stacking faults theoretically by using molecular dynamics simulations. Three interaction modes between screw dislocation and SF, and three interaction modes between screw dislocation and ESF, have been identified by using MD simulations in FCC metals. The six interaction modes can be classified into two categories-one is cross slip (modes 1, 2, 4, and 5), the other is penetration (modes 3 and 6 ). Both the two categories have been observed experimentally in dislocation-twin interaction $[1,37,38]$. Since stacking faults are also basic planar defects as well as twin boundary, the reaction of screw dislocation at the stacking faults may be similar in many ways.

According to our simulation results, the occurrence of cross slip is possible for screw dislocation during its reaction with stacking fault when the following conditions are met:

(1) Cross slip is not possible for all the FCC metals (see Table 1). It depends on the stacking fault energy, the unstable stacking fault energy and the twin boundary migration energy.

(2) The resolved shear stress should be both larger than CRSS for the combination of dislocation before SF/ESF, and smaller than CRSS for the penetration of SF/ESF (see Table 1). 
(3) When the dislocation reaches the stacking fault plane, the dislocation line should become parallel to the intersection line of the slip plane and the stacking fault plane. As to our investigation, it also means that the Burgers vector of the lattice screw dislocation should be parallel to the pre-existing stacking fault plane.

We analyze the resistance to, and establish the criterion for, each mode. It is revealed that the dimensionless parameters, relevant to both planar fault energies and their ratios, can be used to predict the propensity to different interaction modes. During the plastic deformation of FCC polycrystalline metals, both dislocations and stacking faults generate continuously, accompanied by the complex stress state. Once the local stress state meets with the criterion for a certain mode, the corresponding interaction will occur.

We obtain the critical resolved shear stresses for the transition among different modes by using MD simulations. Similar to the strengthening mechanism of coherent twin boundaries studied before [15,39], SF and ESF, as obstacles to dislocations, may both strengthen metals too, due to the stress concentration resulted from the recombination of extended dislocation and the penetration process, although the strengthening effect may be less abundant. The screw dislocations after penetrating SF/ESF are still glissile ones, which can take part in the subsequent plastic deformation and do favors to the ductility of materials. And the annihilation of SF has had a positive influence on metal's ductility.

\section{Acknowledgments}

All of the simulations were performed using Lammps Molecular Dynamics Simulator [40] and visualized by Atomeye [41]. This work is supported by National Science Foundation of China through Grants Nos. 11021262, 10932011, 90816004 and 91116003; and by National Basic Research Program of China through 2012CB937500.

\section{Appendix A. Supplementary data}

Supplementary data associated with this article can be found, in the online version, at doi:10.1016/j.msea.2012.01.115.

\section{References}

[1] K. Lu, L. Lu, S. Suresh, Science 324 (2009) 349.
[2] W.D. Callister, Materials Science and Engineering: An Introduction, 7th ed., John Wiley \& Sons, New York, 2007.

[3] D. Hull, D.J. Bacon, Introduction to Dislocations, 4th ed., ButterworthHeinemann, Oxford, 2001.

[4] J.P. Hirth, J. Lothe, Theory of Dislocations, 2nd ed., John Wiley \& Sons, New York, 1982.

[5] R.B. Schwarz, A.V. Granato, Phys. Rev. Lett. 34 (1975) 1174.

[6] C. Eisenmann, U. Gasser, P. Keim, G. Maret, H. von Grunberg, Phys. Rev. Lett. 95 (2005) 185502.

[7] M. Bhattachary, A. Dutta, P. Mukherjee, N. Gayathri, P. Barat, Phys. Rev. B 82 (2010) 184113.

[8] T. Tsuru, Y. Shibutani, Y. Kaji, Phys. Rev. B 79 (2009) 012104.

[9] Z.-H. Jin, P. Gumbsch, E. Ma, K. Albe, K. Lu, H. Hahn, H. Gleiter, Scripta Mater. 54 (2006) 1163.

[10] Z.-H. Jin, P. Gumbsch, K. Albe, E. Ma, K. Lu, H. Gleiter, H. Hahn, Acta Mater. 56 (2008) 1126.

[11] Z.X. Wu, Y.W. Zhang, D.J. Srolovitz, Acta Mater. 57 (2009) 4508.

[12] J. Wang, H.C. Huang, Appl. Phys. Lett. 88 (2006) 203112.

[13] Y. Cheng, M. Mrovec, P. Gumbsch, Philos. Mag. 88 (2008) 547.

[14] L. Lu, X. Chen, X. Huang, K. Lu, Science 323 (2009) 607.

[15] A.J. Cao, Y.G. Wei, S.X. Mao, Appl. Phys. Lett. 90 (2007) 151909.

[16] A.J. Cao, Y.G. Wei, J. Appl. Phys. 102 (2007) 083511.

[17] F.A. Mohamed, T.G. Langdon, J. Appl. Phys. 45 (1974) 1965.

[18] C.R. Barrett, O.D. Sherby, Trans. Am. Inst. Min. Eng. 233 (1965) 1116.

[19] F.A. Mohamed, Acta Mater. 51 (2003) 4107.

[20] V. Yamakov, D. Wolf, S.R. Phillpot, H. Gleiter, Acta Mater. 51 (2003) 4135.

[21] P. Szelestey, M. Patriarca, K. Kaski, Modell. Simul. Mater. Sci. Eng. 13 (2005) 541.

[22] H.J. Lee, J.H. Shim, B.D. Wirth, J. Mater. Res. 22 (10) (2007) 2758.

[23] Y.N. Osetsky, R.E. Stoller, Y. Matsukawa, J. Nucl. Mater. 329 (2004) 1228.

[24] Y. Matsukawa, M. Briceno, I.M. Robertson, Microsc. Res. Tech. 72 (3) (2009) 284.

[25] Y.T.Zhu, X.L. Wu, X.Z. Liao, J. Narayan, L.J. Kecskes, S.N. Mathaudhu, Acta Mater. $59(2011) 812$.

[26] G. Grochola, S.P. Russo, I.K. Snook, J. Chem. Phys. 123 (2005) 204719.

[27] P.L. Williams, Y. Mishin, J.C. Hamilton, Modell. Simul. Mater. Sci. Eng. 14 (2006) 817.

[28] Y. Mishin, D. Farkas, M.J. Mehl, D.A. Papaconstantopoulos, Phys. Rev. B 59(1999) 3393.

[29] Y. Mishin, M.J. Mehl, D.A. Papaconstantopoulos, A.F. Voter, J.D. Kress, Phys. Rev. B 63 (2001) 224106.

[30] J.R. Rice, J. Mech. Phys. Solids 40 (1992) 239.

[31] S. Hai, E.B. Tadmor, Acta Mater. 51 (2003) 117

[32] E.B. Tadmor, S. Hai, J. Mech. Phys. Solids 51 (2003) 765.

[33] H.V. Swygenhoven, P.M. Derlet, A.G. Frøseth, Nat. Mater. 3 (2004) 399

[34] A.G. Frøseth, H.V. Swygenhoven, P.M. Derlet, Acta Mater. 52 (2004) 2259.

[35] Q.Y. Hu, L. Lan, N.M. Ghoniem, Acta Mater. 57 (2009) 4866.

[36] J.A. Zimmerman, H.J. Gao, F.F. Abraham, Modell. Simul. Mater. Sci. Eng. 8 (2000) 103.

[37] Y.B. Wang, M.L. Sui, Appl. Phys. Lett. 94 (2009) 021909.

[38] M. Dao, L. Lu, Y.F. Shen, S. Suresh, Acta Mater. 54 (2006) 5421

[39] C. Deng, F. Sansoz, Nano Lett. 9 (2009) 1517.

[40] S.J. Plimpton, J. Comput. Phys. 117 (1995) 1.

[41] J. Li, Modell. Simul. Mater. Sci. Eng. 11 (2003) 173. 